\title{
Transorbital penetrating cerebral injury with a ceramic stone: Report of an interesting case
}

\author{
G. D. Satyarthee, Sachin A. Borkar, Anuj Kumar Tripathi, B. S. Sharma \\ Department of Neurosurgery, Jai Prakash Narayan Apex Trauma Center, All India Institute of Medical Sciences, New Delhi, India
}

\author{
Address for correspondence: \\ Dr. G. D. Satyarthee, \\ Department of Neurosurgery, \\ All India Institute of Medical \\ Sciences, Ansarinagar, \\ New Delhi - 110 029, India. \\ E-mail: duttaguru2002@yahoo.com
}

DOI : 10.4103/0028-3886.53285

\begin{abstract}
Penetrating cranial injury is a potentially life-threatening condition. The majority of war injuries are high-velocity penetrating cranial injuries; but in civilian cases, most penetrating cranial wounds are low-velocity type. We report an interesting case of transorbital penetrating cranial injury with a knife-sharpening stone made up of ceramic in a 28-yearold male. The pertinent literature is reviewed and management of such cases is discussed.
\end{abstract}

Key words: Head injury, penetrating head injury, transorbital

\section{Introduction}

Although head injuries are a common cause of accidental trauma in all age groups, penetrating head trauma is rare. Penetrating head injury (PHI) in civilian population is mostly low-velocity type caused by knives, nails, spikes, iron rods, pencils, scissors, keys, underwater fishing harpoon, fan blades, and screwdrivers. Authors report one such rare case of transorbital penetrating cranial injury caused by a ceramic stone, and discuss the implications and management of such rare type of injury.

\section{Case Report}

A 28-year-old male presented to our emergency services in altered sensorium after an accident at his workplace. The patient was a construction worker and history revealed that a piece of knife-sharpening stone, fixed on an electric revolving system, broke during work and entered in to the head through right orbital roof. On physical examination, the patient had a pulse rate of 110 per minute and blood pressure of 136/80 $\mathrm{mm} \mathrm{Hg}$. His Glasgow Coma Scale (GCS) score, on admission, was 8. Left pupil was normal in size and reacting to light, whereas right eye globe was ruptured. There was a lacerated wound over the right side of his forehead extending to right supraorbital margin. The brain matter was herniating through the wound. [Figure 1]. X-rays of the chest and cervical spine were normal. Focused abdominal sonogram for trauma (FAST) was also negative. The patient was immediately intubated and put on ventilator.

Computerized tomography (CT) scan of the head revealed a foreign body in right parietal region with depressed fracture of the right frontal bone and right orbital roof extending to right medial orbital wall and the ethmoid. There was right frontotemporal acute subdural hematoma with bifrontal contusion and pneumocephalus. Basal cisterns were effaced and significant midline shift was present toward left side. Trajectory of the foreign body was extending from right orbital roof to right parietal region [Figures 2 and 3].

The patient was immediately shifted to operating room for emergency exploration. Right frontotemporoparietal skin flap and osteoplastic bone flap were raised. Dural tear was present in right frontal region extending to basifrontal region and the brain matter was herniating through it. The dural flap based on superior sagittal sinus was raised and the foreign body was removed along with evacuation of acute subdural hematoma. Contused necrotic brain surrounding the foreign body and depressed bony fragments were also removed [Figure 4]. Hemostasis was achieved and dura repaired with pericranium and temporalis fascia. Enucleation of the right eyeball was done simultaneously by ophthalmic surgeon. A subdural catheter was placed prior to wound closure. He was 


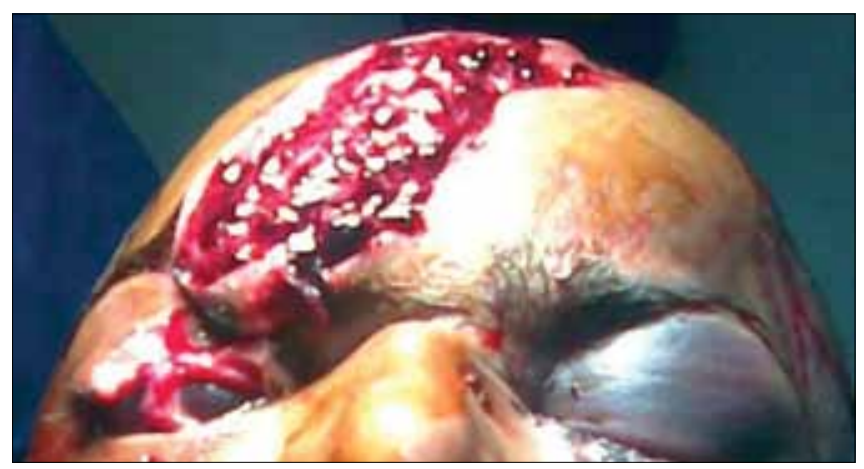

Figure 1: Clinical photograph showing lacerated wound extending from right supraorbital margin to right parietal region with herniation of the brain tissue with right eye globe rupture

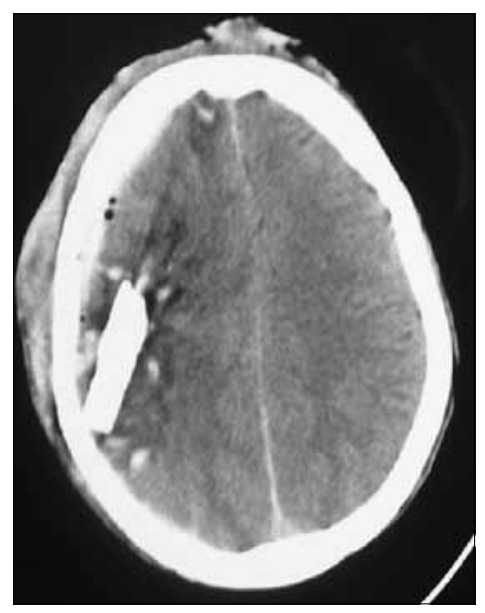

Figure 3: NCCT of the head showing trajectory of the foreign body, extending from right orbital roof to right parietal region associated with contusion in surrounding brain parenchyma along with traumatic subarachnoid hemorrhage

prescribed decongestants (mannitol, frusemide) along with antiepileptic (phenytoin) and antibiotics during the postoperative period. He was electively ventilated for 72 hours, gradually weaned off, and extubated. The intracranial pressure (ICP) was monitored in the postoperative period for three days and was within normal limits. Postoperative course was uneventful and the patient was discharged on postoperative day 11 . At the time of discharge, the patient's neurological response was E3V2M5 $(\mathrm{GCS}=10)$. The patient recovered in due course of time and recovered to GCS of 15 at six-month follow-up visit.

\section{Discussion}

Penetrating head injuries have higher mortality and morbidity than blunt trauma even in civilian set up. Case fatality rates are higher for penetrating than closed injuries for all GCS, gender, age, and cause of injury categories. PHI can be either high- or low-velocity type. Worldwide, most PHIs are caused by missile injuries, notably gun shot and shrapnel wounds. Most nonmissile PHIs are caused by knives or cutting instruments but the list of

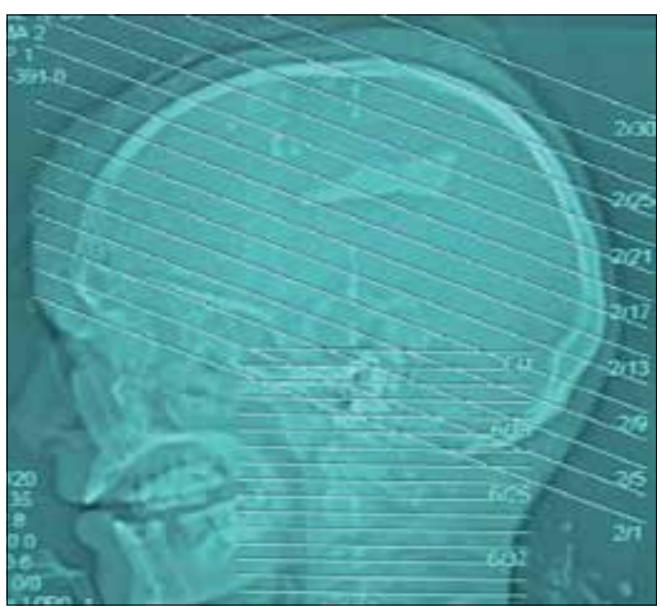

Figure 2: CT topogram showing the foreign body (ceramic stone) lodged in parietal region

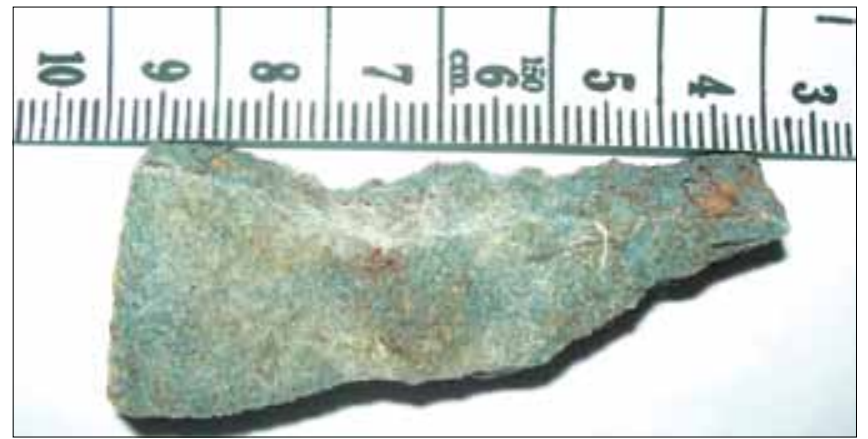

Figure 4: Figure showing extracted foreign body (ceramic stone)

other objects includes stones, sharpened wooden sticks, screwdrivers, nails, spikes, iron rods, arrows, pencils, ice picks, chopsticks, umbrella ends, thumbtacks, fishing harpoons, paint brushes, scissors, crotchet hooks, sewing needles, and garden forks. ${ }^{[1,2,3]}$

In civilian accidents, most penetrating cranial wounds are of low-velocity type caused by knives, nails, spikes, iron rods, pencils, scissors, keys, underwater fishing harpoon, fan blades, and screwdrivers. ${ }^{[1]}$ Ceramic sharpening stones are produced by kiln-firing gritty particles with a ceramic bonding agent. Because they are manmade, ceramic stones tend to have greater accuracy in the grit rating. Additionally they use metal particles such as alumina or steel, which makes them last longer than naturally occurring one.

In cranial vault, orbital roof and temporal bone are relatively thinner parts so that projectile objects with less force can easily penetrate and travel long distance inside the brain parenchyma through these corridors. ${ }^{[4]}$ In our case, a triangular piece of revolving stone penetrated the right orbital roof and reached up to right parietal region.

The pathophysiological consequences of penetrating head wounds depend on the kinetic energy and the trajectory 
of the object through the brain. Lower velocity objects produce a track of primary tissue damage, resulting in a focal, localized brain parenchymal injury without diffuse or global affects. In the present case, contused brain was present around the stone, depressed bony segments and, along the trajectory. In contrast, higher velocity objects result in extensive brain injury caused by cavitation and the rapid transfer of kinetic energy into the surrounding tissues. ${ }^{[5]}$ Also, the degree of permanent neurological damage depends upon severity of the injury, location (eloquent versus noneloquent cortex), and duration from injury to operative intervention (early versus delayed).

Computed tomography scan is useful in the initial evaluation because it can readily determine the extent of intraparenchymal injury, locate the offending object, plot its trajectory, and identify most bony defects in the skull.

The consequences of penetrating head wounds include intraparenchymal lesions like cerebral contusions, cerebrospinal (CSF) fluid fistulas, intracerebral hematoma, subdural and epidural hematoma, subarachnoid hemorrhage, pneumocephalus, skull fracture, cerebral edema, etc. Infectious complications include brain abscess, encephalitis, meningitis, and scalp sepsis. Vascular complications like pseudoaneurysm, post-traumatic arteriovenous malformation, though rare, can occur following PHI. ${ }^{[6]}$ Diffuse subarachnoid hemorrhage, as in our case, is known to be associated with a poor outcome. ${ }^{[7]}$

Initial emergency treatment of PHIs includes the usual airway and circulatory resuscitation as well as local hemostasis. ${ }^{[2]}$ Because these injuries are by definition compound fractures, tetanus toxoid and prophylactic broad-spectrum antibiotics amenable to crossing the blood-brain barrier should be given early in patient management. Expeditious removal of the penetrating foreign body and bone fragments along with focal debridement of the scalp, skull, dura, and involved parenchyma are the goals of surgical treatment. Watertight dural closure has long been a mainstay of the surgical management of penetrating brain injuries and serves to prevent cerebral fungus and reduce the instance of CSF leak. Dura can be repaired either primarily if possible or with dural patch harvested from pericranium or temporalis fascia. ${ }^{[2]}$

Degree of permanent neurologic deficit associated with low-velocity penetrating skull wounds is determined by the degree and location of the initial injury, the rapidity of operative exploration and debridement, and the avoidance of delayed secondary injury. One should pay close attention to basic perioperative and intraoperative principles in managing such frequently fatal injuries in order to provide a satisfactory functional outcome.

\section{References}

1. Seex K, Koppel D, Fitzpatrick M, Pyott A. Trans-orbital penetrating head injury with a door key. J Craniomaxillofac Surg 1997;25:353-5.

2. Kelly DF, Nikas DL, Becker DP. Diagnosis and treatment of moderate and severe head injuries in adults. In Youmans JR, ed. Neurological Surgery. $4^{\text {th }}$ ed. Philadelphia: WB Saunders; 1996. p. 1618-718.

3. Bhaganagare A, Nadkarni T, Goel A. Penetrating craniocerebral injury with nails: Case report. Indian Journal of Neurotrauma (IJNT) 2007;4:63-4.

4. Gopalakrishnan MS, Devi IB. Fatal penetrating orbitocerebral injury by bicycle brake handle. Indian Journal of Neurotrauma (IJNT) 2007;4:123-4.

5. Harsh G R. Penetrating wounds of the head. In: Williams, Rengachary, editors. Neurosurgery. New York: McGraw-Hill; 1985. p. 1670-78.

6. Peek-Asa C, McArthur D, Hovda D, Kraus J. Early predictors of mortality in penetrating compared with closed brain injury. Brain Inj 2001;15:801-10.

7. Kieck CF, de Villiers JC. Vascular lesions due to transcranial stab wounds. J Neurosurg 1984;60:42-6.

Accepted on 16-03-2009

Source of Support: Nil, Conflict of Interest: None declared. 